\title{
Semiquantitative mIBG Scoring as a Prognostic Indicator in Patients with Stage 4 Neuroblastoma: A Report from the Children's Oncology Group
}

\author{
Gregory A. Yanik ${ }^{1}$, Marguerite T. Parisi ${ }^{2,3}$, Barry L. Shulkin ${ }^{4}$, Arlene Naranjo ${ }^{5}$, Susan G. Kreissman ${ }^{6}$, Wendy B. London ${ }^{7}$, \\ Judith G. Villablanca ${ }^{8}$, John M. Maris ${ }^{9}$, Julie R. Park ${ }^{3}$, Susan L. Cohn ${ }^{10}$, Patrick McGrady ${ }^{5}$, and Katherine K. Matthay ${ }^{11}$ \\ ${ }^{I}$ Department of Pediatrics, University of Michigan Medical Center, Ann Arbor, Michigan; ${ }^{2}$ Department of Radiology, Seattle \\ Children's Hospital/University of Washington School of Medicine, Seattle, Washington; ${ }^{3}$ Department of Pediatrics, Seattle Children's \\ Hospital/University of Washington School of Medicine, Seattle, Washington; ${ }^{4}$ Department of Radiological Sciences, St. Jude \\ Children's Research Hospital, Memphis, Tennessee; ${ }^{5}$ Children's Oncology Group, University of Florida, Gainesville, Florida; \\ ${ }^{6}$ Department of Pediatrics, Duke University Medical Center, Durham, North Carolina; ${ }^{7}$ Children's Oncology Group, Boston/Dana- \\ Farber Cancer Institute, Boston, Massachusetts; ${ }^{8}$ Department of Pediatrics, Children's Hospital Los Angeles/University of Southern \\ California Keck School of Medicine, Los Angeles, California; ${ }^{9}$ Department of Pediatrics, Children's Hospital of Philadelphia and \\ University of Pennsylvania, Philadelphia, Pennsylvania; ${ }^{10}$ Department of Pediatrics, University of Chicago, Chicago, Illinois; and \\ ${ }^{11}$ Department of Pediatrics, University of California San Francisco, San Francisco, California
}

Radiolabeled metaiodobenzylguanidine (mIBG) is a highly sensitive and specific marker for detecting neuroblastoma. A semiquantitative mIBG score (Curie score [CS]) was assessed for utility as a prognostic indicator for a cohort of patients with highrisk metastatic disease. Methods: mIBG scans from 280 patients with mIBG-avid, stage 4 neuroblastoma enrolled on the Children's Oncology Group (COG) protocol A3973 were evaluated at diagnosis $(n=280)$, after induction chemotherapy $(n=237)$, and after an autologous stem cell transplantation $(n=178)$. Individual mIBG scans were evaluated at 10 different anatomic regions, with the scoring of each site (0-3) based on the extent of disease at that anatomic region. Results: There was no correlation between CS at diagnosis and subsequent treatment outcome. Patients with a CS $>2$ after induction therapy had a significantly worse event-free survival (EFS) than those with scores $\leq 2$ (3-y EFS: $15.4 \% \pm 5.3 \%$ vs. $44.9 \% \pm 3.9 \%$, respectively; $P<0.001$ ). A postinduction CS $>2$ identified a cohort of patients at greater risk for an event, independent of other known neuroblastoma factors, including age, $M Y C N$ status, ploidy, mitosiskaryorrhexis index, and histologic grade. For MYCN-amplified tumors, the presence $(C S>0)$ versus absence $(C S=0)$ of residual $\mathrm{mIBG}$ avidity after induction was associated with a significantly worse outcome (3-y EFS: $11.8 \% \pm 7.8 \%$ vs. $49.6 \% \pm 7.7 \%$, respectively; $P=0.003$ ). After transplantation, patients with a CS $>0$ had an EFS inferior to that of patients with a CS of 0 (3-y EFS: $28.9 \% \pm 6.8 \%$ vs. $49.3 \%$ $\pm 4.9 \%$, respectively $[n=133] ; P=0.009$ ). Conclusion: Curie scoring carries prognostic significance in the management of patients with high-risk neuroblastoma. In particular, patients with CSs $>2$ after induction have extremely poor outcomes and should be considered for alternative therapeutic strategies.

Received Sep. 6, 2012; revision accepted Nov. 5, 2012.

For correspondence or reprints contact: Gregory Yanik, University of Michigan Medical Center, 1500 E. Medical Center Dr., Ann Arbor, MI 48109. E-mail: gyanik@umich.edu

Published online Feb. 25, 2013.

COPYRIGHT @ 2013 by the Society of Nuclear Medicine and Molecular Imaging, Inc.
Key Words: neuroblastoma; mIBG; pediatrics; bone marrow transplantation

J Nucl Med 2013; 54:541-548

DOI: 10.2967/jnumed.112.112334

$\mathbf{O}$ utcomes for patients with stage 4 high-risk neuroblastoma have been historically poor, with 5-y disease-free survival rates of only $30 \%-46 \%$ for patients older than 1 y (1-3). Despite multimodal therapy, durable remission rates remain low, with $10 \%-15 \%$ of patients progressing during induction therapy and another $40 \%$ progressing after an initial response to induction $(2,3)$. The addition of autologous stem cell transplantation (ASCT) after induction therapy, improved surgical and radiotherapy techniques, and the recent use of a chimeric anti-GD2 antibody after transplantation have all led to incremental improvements in disease-free survival (4). The ability to identify both clinical and biologic prognostic markers of response early in a patient's clinical course may significantly affect subsequent therapy, identifying individuals who may benefit from augmented or alternative therapy.

Metaiodobenzylguanidine (mIBG) is an aralkylguanidine with structural similarity to the catecholamine norepinephrine (5). Scintigraphic studies in the 1980s confirmed the ability of radiolableled mIBG to localize both pheochromocytoma $(6,7)$ and neuroblastoma $(8-11)$. Radioiodination studies with ${ }^{131} \mathrm{I}$ and ${ }^{123} \mathrm{I}$ have been performed, with both ${ }^{131} \mathrm{I}$-mIBG and ${ }^{123} \mathrm{I}-\mathrm{mIBG}$ currently licensed for scintigraphic imaging of neuroblastoma. Approximately $90 \%$ of neuroblastomas concentrate either ${ }^{123} \mathrm{I}-\mathrm{mIBG}$ or ${ }^{131} \mathrm{I}$-mIBG within marrow, cortical bone, or soft-tissue sites of disease (11-13). ${ }^{123} \mathrm{I}-$ and ${ }^{131} \mathrm{I}$-mIBG uptake has been noted in patients with low- or advanced-stage disease, favorable or 
unfavorable histologic patterns, amplified or nonamplified MYCN gene status, and elevated or normal catecholamine levels (11).

Previous studies have indicated that mIBG uptake may be a useful surrogate marker for evaluating response to therapy (14-21). Initial reports focused on the qualitative features of mIBG scans, primarily noting whether disease was detectable. Over the past $15 \mathrm{y}$, a semiquantitative scoring system (Curie scoring) has been developed to predict the extent and severity of mIBG-avid disease (14-16). The Curie scoring system has been reproducible, with good intra- and interobserver concordance (16). The presence of mIBG-avid disease immediately before myeloablative therapy has been associated with subsequent disease progression using these criteria $(15-17,20,22)$. However, these studies were often limited by small sample sizes or heterogeneous study populations and therapy regimen $(16,22)$.

The aim of our current study was to investigate the use of the Curie scoring system as a prognostic marker of response and survival for patients with mIBG-avid, stage 4 newly diagnosed high-risk neuroblastoma, treated on the recent Children's Oncology Group (COG) protocol A3973. The determination of an optimal cut-point with respect to survival at each time point-at diagnosis, after induction, and after transplantation-is examined.

\section{MATERIALS AND METHODS}

\section{Patient Population}

Three hundred nine patients with newly diagnosed stage 4 (per International Neuroblastoma Staging System) (23) high-risk neuroblastoma who were enrolled on COG protocol A3973, had not received prior systemic therapy, and had undergone mIBG scans at diagnosis were eligible for analysis. Of these 309 patients, 280 (90.6\%) exhibited mIBG-avid disease at diagnosis and were subsequently included in this analysis. The 29 patients with non-mIBG-avid disease at diagnosis were excluded from postdiagnosis outcome analysis. All patients had undergone an ${ }^{123} \mathrm{I}-$ or ${ }^{131}$ I-mIBG scan at diagnosis, with subsequent scans obtained after induction $(n=237)$ or after ASCT $(n=178)$ (Table 1$)$. Written informed consent was obtained from all patients (or legal guardians) before study entry.

\section{Treatment}

Treatment consisted of 6 cycles of induction chemotherapy, surgical resection of residual soft-tissue disease, autologous peripheral blood stem cell transplantation (ASCT), radiotherapy, and biotherapy (Fig. 1). Patients were randomized at study entry to undergo purged or unpurged autologous stem cell collection after the second cycle of induction therapy, with subsequent ASCT after the sixth induction cycle. Tumor cell purging of peripheral blood stem cells was performed using immunomagnetic beads in a central laboratory. Radiotherapy guidelines for the primary and metastatic sites were specified, including the total dose administered and fractionation. Patients subsequently received 6 monthly cycles of isotretinoin beginning day +66 after transplantation. In addition, 46 patients were enrolled onto COG ANBL0032 and treated with chimeric anti-GD2 antibody plus isotretinoin (4).
TABLE 1

Patient Characteristics at Diagnosis

\begin{tabular}{|c|c|}
\hline Characteristic & $n$ \\
\hline Total patients ${ }^{\star}$ & 280 \\
\hline \multicolumn{2}{|l|}{ Sex } \\
\hline Male & $165(59)$ \\
\hline Female & $115(41)$ \\
\hline Age $<18 \mathrm{mo}$ & $31(11)$ \\
\hline Age $>18 \mathrm{mo}$ & $249(89)$ \\
\hline \multicolumn{2}{|l|}{ Disease site ${ }^{\dagger}$} \\
\hline Bone only & $0(0)$ \\
\hline Bone marrow only & $2(1)$ \\
\hline Soft tissue only & $49(18)$ \\
\hline Bone and bone marrow & $3(1)$ \\
\hline Bone and soft tissue & $22(8)$ \\
\hline Bone marrow and soft tissue & $29(10)$ \\
\hline Bone, bone marrow, and soft tissue & $174(62)$ \\
\hline Unknown & 1 \\
\hline \multicolumn{2}{|l|}{ MYCN status } \\
\hline Amplified & $85(38)$ \\
\hline Nonamplified & $140(62)$ \\
\hline Unknown & 55 \\
\hline \multicolumn{2}{|l|}{ Tumor histology } \\
\hline Favorable & $6(3)$ \\
\hline Unfavorable & $188(97)$ \\
\hline Unknown & 86 \\
\hline \multicolumn{2}{|l|}{ Ploidy } \\
\hline Hyperdiploid & $103(47)$ \\
\hline Diploid & $116(53)$ \\
\hline Unknown & 61 \\
\hline \multicolumn{2}{|l|}{ mIBG scans scored } \\
\hline Diagnosis & 280 \\
\hline After induction & 237 \\
\hline After ASCT & 178 \\
\hline
\end{tabular}

*Includes only patients with mIBG-avid disease $(n=280)$ present at diagnosis.

${ }^{\dagger}$ Indicates sites of disease at diagnosis, as determined by radiographic studies (CT/MRI, mIBG scanning), soft-tissue biopsy or bone marrow aspirate/biopsy.

Data in parentheses are percentages. Median age was $3.1 \mathrm{y}$, and age range was $0.6-29 \mathrm{y}$.

\section{Diagnostic Imaging: mIBG Scans}

Diagnostic imaging was performed with either ${ }^{123} \mathrm{I}-\mathrm{mIBG}$ or ${ }^{131} \mathrm{I}$-mIBG scanning techniques $(11,24)$. For thyroid protection, supersaturated potassium iodide was typically begun $24 \mathrm{~h}$ before the diagnostic mIBG dose and subsequently continued for 3-7 d. ${ }^{123} \mathrm{I}$-mIBG or ${ }^{131} \mathrm{I}$-mIBG planar images, with or without tomography, were acquired using recommended guidelines $(11,24)$.

\section{mIBG Semiquantitative Scoring: Modified Curie Scoring Method}

mIBG scoring was performed using a modified Curie scoring system. Initially developed in 1995, scoring is based on the presence of mIBG uptake in multiple anatomic regions $(14,15)$. Ten different sites were scored, including 9 skeletal sites (head, chest, T-spine, L-spine, pelvis, upper arms, lower arms, femurs, and lower legs) and an additional tenth site for soft-tissue lesions (Fig. 2A). Skeletal sites were individually scored from 0 to 3 as follows: 0 , no mIBG involvement; 1 , one mIBG-avid lesion present; 2 , more than one mIBG-avid lesion present; and 3 , mIBG avidity present 


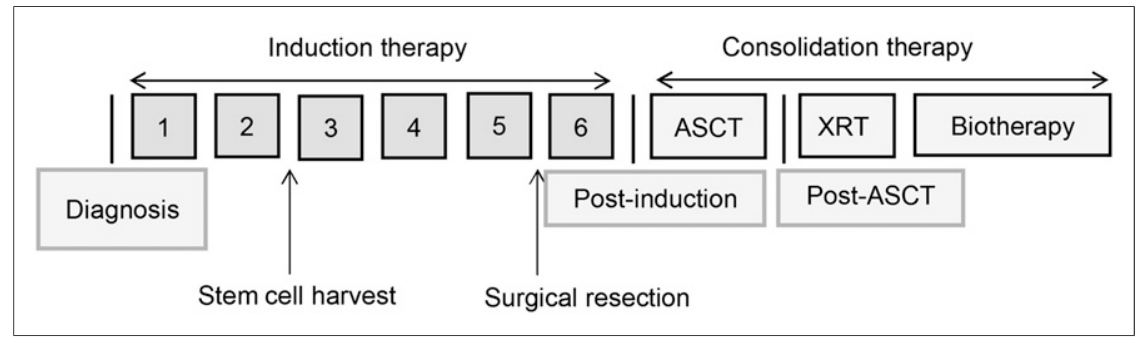

in $>50 \%$ of an individual site. Soft-tissue lesions were scored as follows: 0 , no mIBG involvement; 1 , one $\mathrm{mIBG}$-avid soft-tissue lesion present; 2 , more than one mIBG-avid soft-tissue lesion present; and 3, mIBG avidity in a soft-tissue lesion that occupied $>50 \%$ of the chest or abdomen. A patient's Curie score at each time point was calculated as the sum of scores over all individual sites, with a maximum score of 30 (Fig. 2B). All mIBG scans were centrally reviewed by 2 nuclear medicine physicians, without knowledge of original scan reports, CT scan results, or other clinical information.

\section{Statistical Analysis}

Patients were categorized by Curie score ( 0 vs. $>0 ; \leq$ optimum cutoff vs. $>$ optimum cutoff) and compared with respect to survival at each time point. The optimum cutoff was determined by maximizing the Youden index with respect to how well the Curie score differentiated patients who had and did not have an event, defined as relapse, progressive disease, secondary malignancy, or death. For instance, a patient with an event with a Curie score above a particular cutoff value would be correctly categorized whereas a patient with an event and a Curie score below the cutoff value would be incorrectly categorized. The Youden index is the maximum of (sensitivity + specificity -1 ) over all threshold values of the Curie score and allows evaluation of a diagnostic test with respect to its true-positive and true-negative rates $(25,26)$.

Survival comparisons were drawn at each individual site by score ( 0 vs. $>0$ only) and for patients with $M Y C N$-amplified and
FIGURE 1. Overview of COG A3973 therapy. mIBG scans were obtained at 3 time points: (1) at diagnosis, (2) after induction, and (3) after ASCT. Study therapy: induction cycles 1, 2, 4, and 6 (cyclophosphamide, doxorubicin, and vincristine); induction cycles 3 and 5 (cisplatin and etoposide). ASCT (carboplatin, etoposide, and melphalan). Radiotherapy (XRT) to primary and metastatic sites, beginning no sooner than $28 \mathrm{~d}$ after ASCT. Biotherapy: isotretinoin given for 14 consecutive days in 28-d cycle (6 cycles) \pm ch14.18 antibody. -nonamplified tumors. The percentage change in Curie score from diagnostic to postdiagnostic scans for patients with both sets of mIBG scan readings was stratified on the basis of score reduction ( $\geq$ or $<50 \%$ reduction and $\geq$ or $<75 \%$ reduction), with survival compared between groups.

For event-free survival (EFS), time to event was defined as the time from diagnosis until the time of first event, or until the time of last contact if no event occurred. For overall survival, death was the only event considered. At each postdiagnosis time point, time to event was calculated from the date of the mIBG scan. Patients who had an event between the date of diagnosis and the date of postdiagnosis mIBG scan went off-study and hence were not included in the analysis at that time point. Survival analyses were performed using the methods of Kaplan and Meier (27), with standard errors per Peto et al. (28). EFS and overall survival are presented as the rate \pm SE. Survival curves were compared using a log-rank test. The freeze date for the survival data was August 2009.

To determine the independent prognostic strength for survival of the Curie score in the presence of various prognostic factors, including age ( $<18 \mathrm{mo}$ vs. $\geq 18 \mathrm{mo}$ ), MYCN status (nonamplified vs. amplified), and ploidy (hyperdiploid vs. diploid), Cox proportional hazards models with the Efron method of handling tied event times were fit. Shimada histology was not included in the models because it is confounded with age; thus, the component variables mitosis-karyorrhexis index (low or intermediate vs. high) and histologic grade (undifferentiated or poorly differentiated vs.

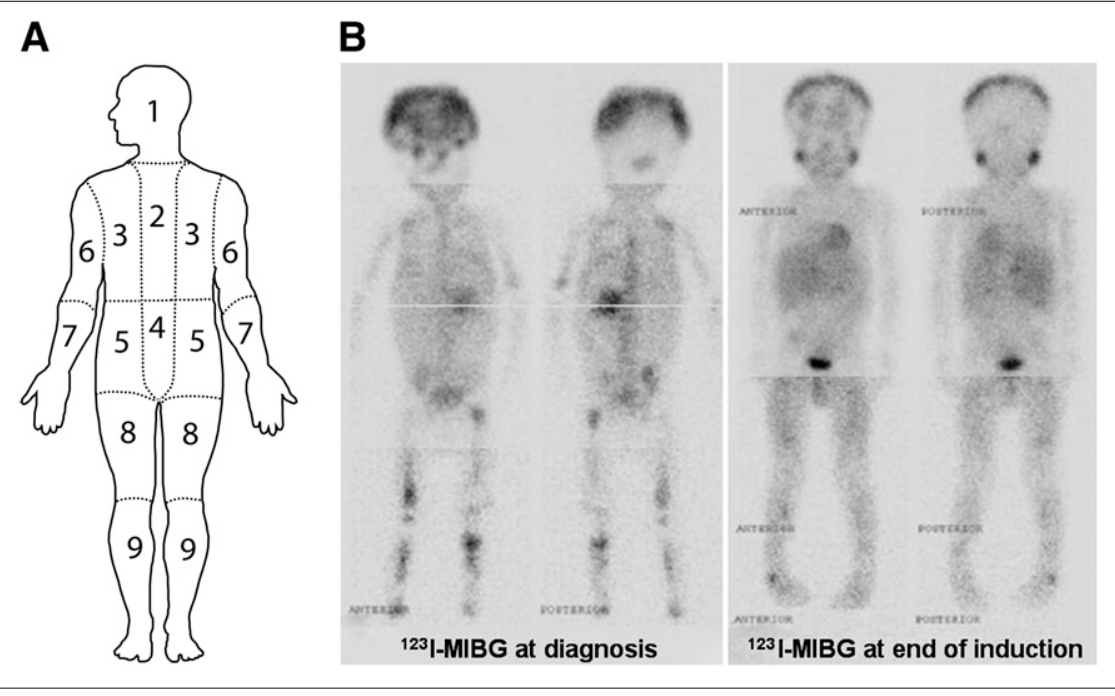

FIGURE 2 (A) Anatomic regions for Curie scoring. (B) Anterior and posterior images from ${ }^{123}$ I-mIBG scans obtained at diagnosis (left) and at end of induction (right) in patient with stage 4, high-risk neuroblastoma. At diagnosis, there was diffuse abnormal ${ }^{123}$ mIBG uptake throughout entire axial and appendicular skeleton (regions 1-9) and uptake in solitary soft-tissue mass, resulting in Curie score of 28 (score $=27$ [ $3 \times 9$ skeletal regions] +1 [soft-tissue region]). After induction, there was decreased but persistent abnormal ${ }^{123}$ I-mIBG uptake resulting in $\mathrm{Cu}-$ rie score of 12 (score $=3$ [region 1] +1 [region 5] +3 [region 6] +3 [region 8] +2 [region 9]). 
differentiating) were included instead. Any violations of the proportional hazards assumption were handled by treating the covariate as time-dependent, accomplished by including a covariate by survival-time interaction term in the model (29). Backward selection was used to determine the most parsimonious model. $P$ values of less than 0.05 were considered statistically significant.

\section{RESULTS}

Characteristics for the 280 patients at diagnosis are shown in Table 1. Six hundred ninety-five mIBG scans were scored: 280 at diagnosis, 237 after induction, and 178 after transplantation. ${ }^{123} \mathrm{I}-\mathrm{mIBG}$ scintigraphy was used in 509 (73\%) scans and ${ }^{131} \mathrm{I}-\mathrm{mIBG}$ in $186(27 \%)$. In $260(93 \%)$ of the 280 patients, the same radioisotope was used for imaging at all time points. No differences were noted in median Curie scores or EFS on the basis of the type of radioisotope used for imaging, allowing us to combine ${ }^{123} \mathrm{I}-$ and ${ }^{131} \mathrm{I}-\mathrm{mIBG}$ scan results for analysis (30). The median Curie score at diagnosis was 12 (range, 1-30); after induction, 0 (range, 0-26); and after transplantation, 0 (range, 0-24).

\section{At Diagnosis}

The 3-y EFS for the 280 patients with mIBG-avid disease (Curie score $>0$ ) at diagnosis was $37.6 \% \pm 3.0 \%$. The optimal cut-point for analyses, as determined by the Youden index, was a Curie score of 9. Three-year EFS was $39.9 \% \pm 4.7 \%$ for patients with a Curie score $\leq 9(n=$ $124)$ versus $35.8 \% \pm 4.0 \%$ for those with a score $>9(n=$ 156) at diagnosis (Fig. 3, $P=0.23$ ). Individual sites of mIBG-avid disease at diagnosis did not affect EFS (data not shown). MYCN status was available in 225 of the 280 cases. When stratified by the presence or absence of $M Y C N$ amplification, EFS was not affected by Curie scores at diagnosis.

The 3-y EFS was significantly higher for the subset of patients $(n=29)$ with non-mIBG-avid disease (Curie score $=0$ ) at diagnosis than for the 280 patients with mIBGavid disease present $(55.0 \% \pm 9.5 \%$ vs. $37.6 \% \pm 3.0 \%$, $P=0.033$ ) (Fig. 4). Patients with non-mIBG-avid disease at diagnosis were excluded from any subsequent analysis.

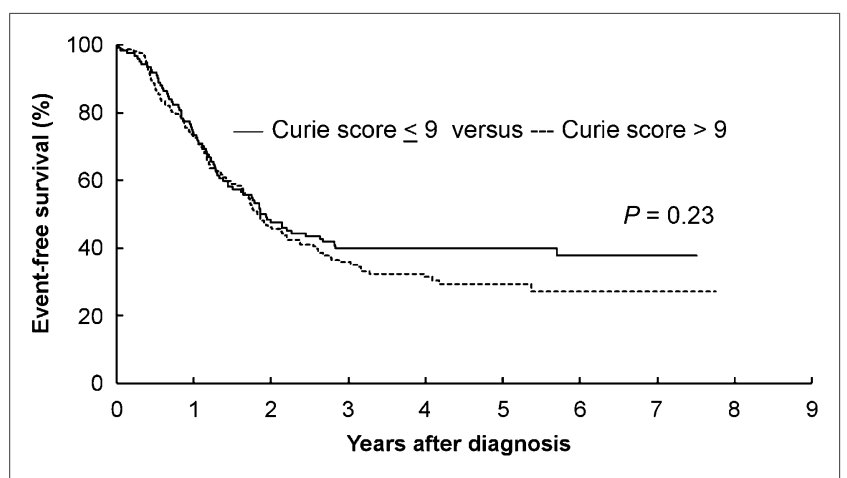

FIGURE 3. EFS by Curie score ( $\leq 9$ vs. $>9)$ at diagnosis $(P=0.23)$, based on Youden index determination of optimal cut-point.

\section{After Induction}

The 3-y EFS for the 237 patients with mIBG scans after induction was $38.3 \% \pm 3.4 \%$. EFS was subsequently determined according to Curie score, with time to event starting from the date of the postinduction mIBG scan. The optimum cut-point after induction, determined by maximizing the Youden index, was a Curie score of 2. Patients with a Curie score $>2(n=52)$ had a significantly decreased EFS in comparison to those with Curie scores $\leq 2(n=185)$ after induction (3-y EFS: $15.4 \% \pm 5.3 \%$ vs. $44.9 \% \pm 3.9 \%$, $P<0.001)$ (Fig. 5A). The presence of any mIBG-avid disease (Curie score $>0, n=93$ ) after induction was also associated with a significantly worse EFS than a Curie score of $0(n=144)$ after induction (3-y EFS: $26.1 \% \pm$ $4.8 \%$ vs. $46.1 \% \pm 4.5 \%, P=0.001)$. Patients with a Curie score of 1 or 2 after induction $(n=41)$ had an EFS similar to that of patients with a Curie score of $0(n=144)$ (3-y EFS: $40.5 \% \pm 8.1 \%$ vs. $46.1 \% \pm 4.5 \%, P=0.40)$. $\mathrm{mIBG}$ avidity isolated to soft-tissue disease alone $(n=23)$ after induction was associated with a $3-y$ EFS of $37.7 \% \pm 10.5 \%$.

The impact of postinduction Curie scores on outcome was examined relative to $M Y C N$ gene status. $M Y C N$ status was available in 191 of the $237(81 \%)$ patients with postinduction mIBG scans, with $M Y C N$ amplification present in 71 of the 191 cases and nonamplification present in 120 . When the optimal cut-point of 2 was used, the 3-y EFS for patients with $M Y C N$-amplified disease was significantly greater for patients with Curie scores $\leq 2(n=65)$ versus $>2(n=6)$ after induction $(44.2 \% \pm 6.9 \%$ vs. $0.0 \%$, $P<0.001$ ). Likewise, the 3-y EFS for patients with non$M Y C N$-amplified disease was significantly greater for those patients with Curie scores $\leq 2(n=90)$ versus $>2(n=30)$ $(50.6 \% \pm 5.5 \%$ vs. $20.8 \% \pm 8.0 \%, P=0.001)$. For patients with $M Y C N$-amplified disease in particular, the presence of any $\mathrm{mIBG}$ avidity (Curie score $>0)$ after induction $(n=17)$ was associated with a significantly worse EFS when compared with patients who were mIBG-negative $(n=54)$ at that time point (3-y EFS: $11.8 \% \pm 7.8 \%$ vs. $49.6 \% \pm 7.7 \%$, $P=0.003$ ) (Fig. 5B).

EFS was affected by the relative change in Curie score, from diagnosis to after induction. Patients with $\geq 50 \%$ reduction in Curie score from diagnosis to after induction $(n=194)$ had significantly improved EFS when compared with those with $<50 \%$ reduction $(n=43)(3$-y EFS: $42.9 \% \pm 3.8 \%$ vs. $17.3 \% \pm 5.9 \%, P<0.001)$. Similar EFS findings were noted when a $\geq 75 \%$ reduction in Curie score was assessed (3-y EFS: $44.4 \% \pm 4.0 \%[n=183]$ vs. $17.4 \% \pm 5.3 \%[n=54], P<0.001)$. However, the presence of a Curie score $>2$ after induction continued to carry prognostic significance, even when taking into consideration the relative change in Curie scores. Survival remained poor for patients with Curie scores $>2$ after induction, regardless of whether a $\geq 50 \%(n=16)$ or $<50 \%$ $(n=36)$ reduction in scores (3-y EFS: $18.8 \% \pm 12.0 \%$ vs. $13.9 \% \pm 5.8 \%, P=0.508$ ) was noted (diagnosis to after induction). Similar results were noted when a $75 \%$ score 


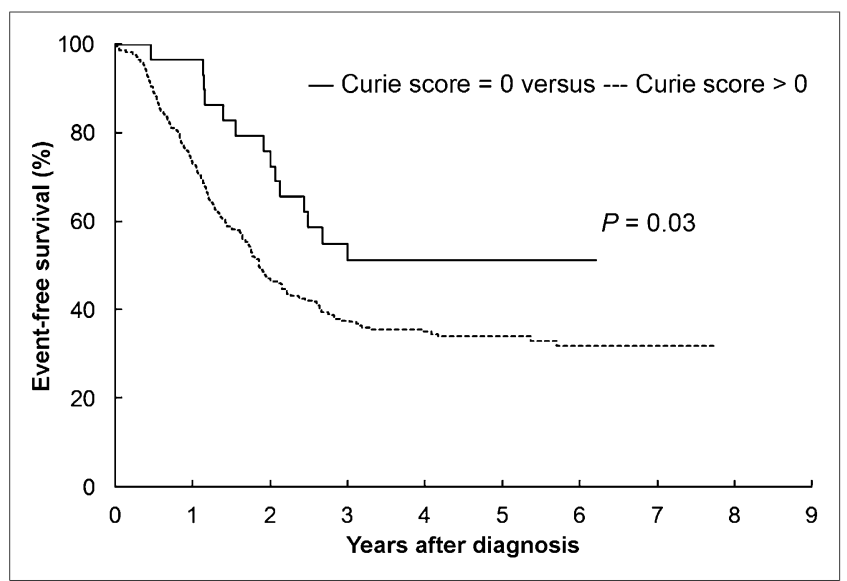

FIGURE 4. EFS for patients with Curie score $(0$ vs. $>0)$ at diagnosis $(P=0.033)$.

reduction $(3-y$ EFS: $22.9 \% \pm 19.6$ vs. $14.0 \% \pm 5.3 \%$, $P=0.571)$ was examined.

A backward-selected Cox model in terms of EFS with a cutoff of 2 after induction indicated that Curie score and ploidy were predictive of EFS, with Curie score values $>2$ and diploid tumors corresponding to an increase in the risk of event of $2.473(n=187 ; P<0.001)$ and 1.538 $(P=0.025)$, respectively. The order of removal was age, histologic grade, mitosis-karyorrhexis index, and MYCN status, with the least statistically significant term dropping out at each step.

\section{After Transplantation}

The optimum cut-point after transplantation, determined by maximizing the Youden index, was a Curie score of 0 . Patients with a Curie score $>0$ after transplantation $(n=45)$ had a significantly worse EFS in comparison to those patients with Curie scores of 0 (3-y EFS: $28.9 \% \pm 6.8 \%$ vs. $49.3 \% \pm 4.9 \%[n=133], P=0.009$ ) (Fig. 6).
MYCN gene status was available in 140 of the $178(79 \%)$ patients with posttransplantation mIBG scans. In contrast to after induction, none of the outcome comparisons by Curie score were statistically significant when stratified by $M Y C N$ status. For patients with $M Y C N$-amplified disease, EFS was similar for patients with Curie scores $>0$ versus 0 (3-y EFS: $37.5 \% \pm 17.1 \%[n=8]$ vs. $45.5 \% \pm 8.7 \%[n=44]$, $P=0.618)$.

Relative scores were also determined according to percentage reduction in Curie score from diagnosis to after transplantation. Only the outcome comparison for EFS with a $\geq 75 \%$ reduction ( $n=152)$ was statistically significant when compared with those with a $<75 \%$ reduction $(n=26)(3$-y EFS: $47.1 \% \pm 4.5 \%$ vs. $26.9 \% \pm 8.7 \%$, $P=0.014)$.

\section{Impact of Chimeric Anti-GD2 Antibody Therapy}

Though all patients received therapy on a uniform protocol (COG A3973), 46 were additionally treated with chimeric anti-GD2 antibody (ch14.18) on COG ANBL0032, on completion of COG A3973 therapy. For patients with Curie scores $\leq 2$ after induction, the 3-y EFS for those patients subsequently treated with chimeric antibody $(n=39)$ was similar to that of the patients $(n=146)$ not receiving antibody (3-y EFS: $48.7 \% \pm 8.0 \%$ vs. $43.9 \% \pm$ $4.5 \%, P=0.526)$. The sample size was too small to identify differences in outcome for patients with Curie scores $>2$ after induction, when based on subsequent treatment with or without chimeric antibody.

\section{DISCUSSION}

In the current study, the quantitative assessment of mIBG scintigraphy after induction chemotherapy was strongly associated with outcome in children with stage 4, mIBGavid, high-risk neuroblastoma. The presence of a Curie score $>2$ after induction therapy was associated with extremely poor survival (15.4\% 3-y EFS) and identified
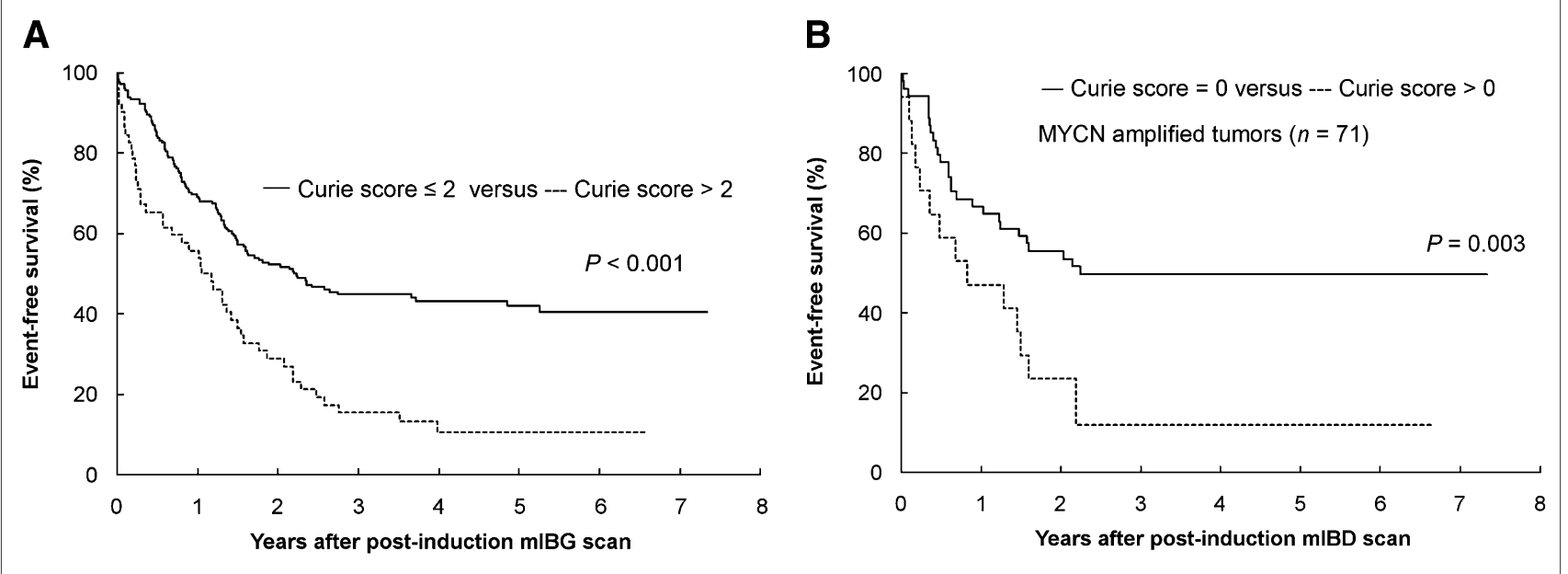

FIGURE 5. (A) EFS by Curie score ( $\leq 2$ vs. $>2)$ after induction $(P<0.001)$, based on Youden index determination of optimal cut-point. (B) EFS by Curie score ( 0 vs. $>0)$ after induction $(P=0.003)$, for patients with $M Y C N$-amplified tumors. 


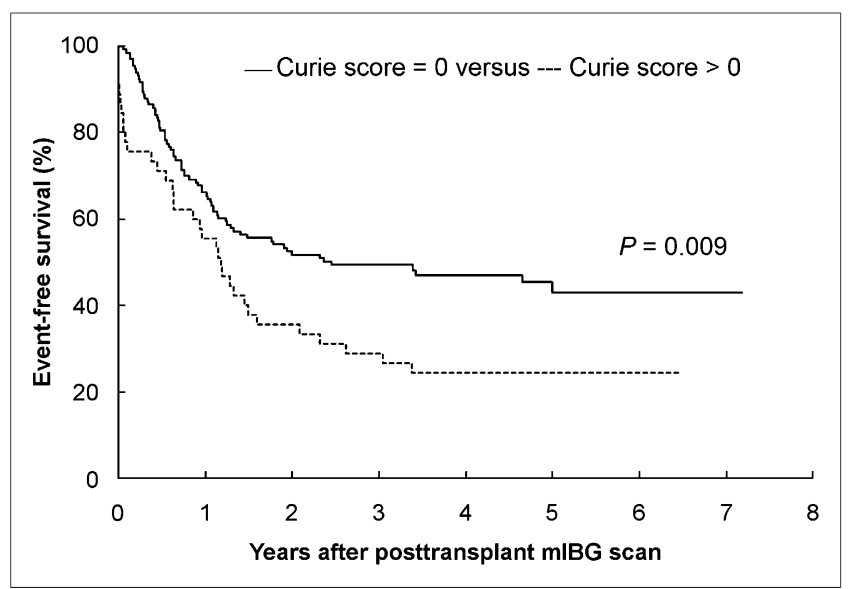

FIGURE 6. EFS by Curie score ( 0 vs. $>0$ ) after transplantation $(P=0.009)$, based on Youden index determination of optimal cut-point.

a cohort of patients at greater risk for an event, independent of other established prognostic markers. Furthermore, patients with $M Y C N$-amplified disease had little benefit from ASCT and subsequent consolidation therapy if they exhibited any mIBG avidity (Curie score $>0$ ) after induction. A beneficial role for ASCT in high-risk neuroblastoma has previously been reported in several cooperative group trials $(1,2,31,32)$. On the basis of our findings, alternative therapy options should be considered for patients with Curie scores $>2$ after induction, including the addition of novel agents before or during consolidation therapy.

Other studies have examined the prognostic impact of mIBG uptake at various time points during therapy, with varying results $(14-22,33)$. Katzenstein et al. noted a similar correlation of elevated Curie score $(>2)$ after induction and EFS in a small series of 29 patients (20). Ladenstein et al., in a large retrospective analysis of the European Bone Marrow Transplant (EBMT) registry, noted that patients with residual mIBG-avid sites after induction had inferior outcomes after subsequent ASCT (22). However, there was significant interpatient variability in therapy in this report, with patients treated over a 14-y period (1978-1992) using a wide range of induction and consolidation regimens. Similar to this EBMT report, we confirmed the finding that patients with residual mIBG-avid disease (Curie score $>0$ ) after induction therapy have inferior outcomes after subsequent consolidation therapy. In comparison to the EBMT report, a strength of our current study is the use of a common induction and consolidation regimen, with patients treated using a single COG high-risk neuroblastoma study.

The effectiveness of anti-GD2 chimeric antibody (ch14.18) in the management of high-risk neuroblastoma has been reported by $\mathrm{Yu}$ et al. (4), with current high-risk neuroblastoma studies advocating ch14.18 as posttransplantation maintenance therapy. Whether the agent is most effective in the management of patients with low tumor burdens (minimal residual disease) is under consideration. In the current analysis, minimal residual disease may be defined as the presence of a low Curie score $(\leq 2)$ after induction. Our data suggest that chimeric antibody did not affect survival in patients with low Curie scores $(\leq 2)$ after induction, with no differences in 3-y EFS identified in those treated with or without chimeric antibody. The data should be interpreted with caution, however, given the retrospective nature of this study and potential patient-investigator bias whether to enroll patients onto COG ANBL0032 (randomized chimeric antibody study) after completion of COG A3973 therapy. The effectiveness of chimeric antibody therapy in patients with low (or high) Curie scores after induction will be examined in future COG studies in which larger populations of patients are treated with chimeric antibody therapy.

Similar to prior reports, non-mIBG-avid disease was noted in $10 \%$ of patients (280/309) with stage 4 disease who were enrolled on COG A3973 (8-13). The improved survival in patients with stage 4 , non-mIBG-avid disease at diagnosis was surprising, with EFS nearly $20 \%$ higher in non-mIBG-avid than mIBG-avid tumors $(P=0.033)$. A formal demographic and outcome comparison for patients treated on COG A3973 with mIBG-negative versus mIBG-positive disease at diagnosis is under examination.

Previous studies have noted that relative scores, determined by the percentage change in Curie score from diagnostic to postdiagnostic scans, carried prognostic significance $(14,15)$. In our current study, however, the impact of relative scores was mitigated in patients who exhibited absolute Curie scores $>2$ after induction, suggesting that the absolute Curie score after induction carried greater prognostic significance than relative scores at that time point. Patients with persistently elevated Curie scores $(>2)$ after induction had inferior outcomes, regardless of whether a $50 \%$ or $75 \%$ reduction in Curie score had been noted. Thus, a patient whose Curie score decreased from 25 to 5 (diagnosis to after induction) could still be projected to have a poor outcome. Historically, International Neuroblastoma Response Criteria have been used to assess response to therapy (23). On the basis of these criteria, for example, a partial response to therapy was historically defined as a $>50 \%$ reduction in the number of mIBG-avid sites, nonresponsive disease a $<50 \%$ reduction in the number of mIBG-avid sites, and progressive disease the development of any new mIBGavid disease. Our current study may ultimately affect future neuroblastoma response definition, with mIBG response potentially defined by an absolute Curie score at specified time points.

The Curie scoring for our study was performed by a central scan review, without knowledge of patient response or outcome. The reproducibility of Curie scoring at local and regional centers will become paramount if 
this scoring method is to be included in risk-group stratification in future clinical trials. A prospective COG study is currently being developed to address this issue.

A prognostic marker is most valuable when effective therapy can be administered using that prognostic marker. For patients with Curie scores $>2$ after induction, current options are limited. The use of therapeutic doses of ${ }^{131} \mathrm{I}$-mIBG as an adjunct to ASCT, either incorporated directly into the ASCT conditioning regimen or administered at a separate time point, has been reported to have therapeutic potential (34-40). To date, therapeutic doses of ${ }^{131} \mathrm{I}-\mathrm{mIBG}$ have been primarily used in patients with refractory or relapsed disease, with response rates of 30\%-40\% noted (39). The use of ${ }^{131} \mathrm{I}-\mathrm{mIBG}$ therapy during induction or consolidation therapy is now a potential option for highrisk patients. Other approaches may include the introduction of novel biologic or immunomodulatory agents that may be active against mIBG-avid, chemotherapy refractory disease.

\section{CONCLUSION}

mIBG scoring is a useful tool to predict outcome for patients with mIBG-avid, stage 4 neuroblastoma. In particular, patients with Curie scores $>2$ after induction may be considered for alternative therapeutic options. The results of these data will undergo validation in upcoming COG highrisk neuroblastoma studies and cross-validation in related European neuroblastoma trials.

\section{DISCLOSURE}

The costs of publication of this article were defrayed in part by the payment of page charges. Therefore, and solely to indicate this fact, this article is hereby marked "advertisement" in accordance with 18 USC section 1734. This study was supported in part by the Elaine and Leland Blatt Foundation and NIH grants U10 CA98413, U10 CA98543, and U10 CA29511. No other potential conflict of interest relevant to this article was reported.

\section{ACKNOWLEDGMENTS}

We thank Thomas J. FitzGerald and staff at the Quality Assurance Review Center (QARC), especially Deirdre Logan, Sandy Kessel, and Fran Laurie, for their tremendous support of this project.

\section{REFERENCES}

1. Philip T, Ladenstein R, Lasset C, et al. 1070 myeloablative megatherapy procedures followed by stem cell rescue for neuroblastoma: 17 years of European experience and conclusions. European Group for Blood and Marrow Transplant Registry Solid Tumour Working Party. Eur J Cancer. 1997;33: 2130-2135.

2. Matthay KK, Villablanca JG, Seeger RC, et al. Treatment of high-risk neuroblastoma with intensive chemotherapy, radiotherapy, autologous bone marrow transplantation, and 13-cis-retinoic acid. Children's Cancer Group. N Engl J Med. 1999;341:1165-1173.

3. Kreissman SG, Villablanca JG, Diller L, et al. Response and toxicity to a doseintensive multi-agent chemotherapy induction regimen for high risk neuroblastoma
(HR-NB): a Children's Oncology Group (COG A3973) study. J Clin Oncol. 2007; 25:9505.

4. Yu AL, Gilman AL, Ozkaynak MF, et al. Anti-GD2 antibody with GM-CSF, interleukin-2, and isotretinoin for neuroblastoma. N Engl J Med. 2010;363: 1324-1334.

5. Wieland DM, Mangner TJ, Inbasekaran MN, et al. Adrenal medulla imaging agents: a structure-distribution relationship study of radiolabeled aralkylguanidines. J Med Chem. 1984;27:149-155.

6. Sisson JC, Frager MS, Valk TW, et al. Scintigraphic localization of pheochromocytoma. N Engl J Med. 1981;305:12-17.

7. Shapiro B, Copp JE, Sisson JC, et al. Iodine-131 metaiodobenzylguanidine for the locating of suspected pheochromocytoma: experience in 400 cases. J Nucl Med. 1985;26:576-585.

8. Treuner J, Feine U, Niethammer D, et al. Scintigraphic imaging of neuroblastoma with [131-I]iodobenzylguanidine. Lancet. 1984;1:333-334.

9. Hoefnagel CA, Voute PA, de Kraker J, et al. Radionuclide diagnosis and therapy of neural crest tumors using iodine-131 metaiodobenzylguanidine. J Nucl Med. 1987;28:308-314.

10. Parisi MT, Greene MK, Dykes TM, et al. Efficacy of metaiodobenzylguanidine as a scintigraphic agent for the detection of neuroblastoma. Invest Radiol. 1992; 27:768-773.

11. Shulkin BL, Shapiro B. Current concepts on the diagnostic use of MIBG in children. J Nucl Med. 1998;39:679-688.

12. Lumbroso JD, Guermazi F, Hartmann O, et al. Meta-iodobenzylguanidine (mIBG) scans in neuroblastoma: sensitivity and specificity, a review of 115 scans. Prog Clin Biol Res. 1988;271:689-705.

13. Claudiani F, Stimamiglio P, Bertolazzi L, et al. Radioiodinated metaiodobenzylguanidine in the diagnosis of childhood neuroblastoma. $Q \mathrm{~J}$ Nucl Med. 1995;39:21-24.

14. Ady N, Zucker JM, Asselain B, et al. A new ${ }^{123}$ I-MIBG whole body scan scoring method: application to the prediction of the response of metastases to induction chemotherapy in stage IV neuroblastoma. Eur J Cancer. 1995;31A:256-261.

15. Matthay KK, Edeline V, Lumbroso J, et al. Correlation of early metastatic response by ${ }^{123}$ I-metaiodobenzylguanidine scintigraphy with overall response and event-free survival in stage IV neuroblastoma. J Clin Oncol. 2003;21: 2486-2491.

16. Messina JA, Cheng S-C, Franc B, et al. Evaluation of semi-quantitative scoring system for metaiodobenzylguanidine (mIBG) scans in patients with relapsed neuroblastoma. Pediatr Blood Cancer. 2006;47:865-874.

17. Perel Y, Conway J, Kletzel M, et al. Clinical impact and prognostic value of metaiodobenzylguanidine imaging in children with metastatic neuroblastoma. J Pediatr Hematol Oncol. 1999;21:13-18.

18. Suc A, Lumbroso J, Rubie H, et al. Metastatic neuroblastoma in children older than one year: prognostic significance of the initial metaiodobenzylguanidine scan and proposal for a scoring system. Cancer. 1996;77:805-811.

19. Kushner BH, Yeh SD, Kramer K, et al. Impact of metaiodobenzylguanidine scintigraphy on assessing response of high-risk neuroblastoma to dose-intensive induction chemotherapy. J Clin Oncol. 2003;21:1082-1086.

20. Katzenstein HM, Cohn S, Shore R, et al. Scintigraphic response by ${ }^{123} \mathrm{I}-$ metaiodobenzylguanidine scan correlates with event-free survival in high-risk neuroblastoma. J Clin Oncol. 2004;22:3909-3915.

21. Schmidt M, Simon T, Hero B, et al. The prognostic impact of functional imaging with ${ }^{123} \mathrm{I}$-mIBG in patients with stage 4 neuroblastoma $>1$ year of age on a highrisk treatment protocol: results of the German Neuroblastoma Trial NB97. Eur J Cancer. 2008;44:1552-1558.

22. Ladenstein R, Philip T, Lasset C, et al. Multivariate analysis of risk factors in stage 4 neuroblastoma patients over the age of one year treated with megatherapy and stem-cell transplantation: a report from the European Bone Marrow Transplantation Solid Tumor Registry. J Clin Oncol. 1998;16:953-965.

23. Brodeur GM, Pritchard J, Berthold F, et al. Revisions of the international criteria for neuroblastoma diagnosis, staging, and response to treatment. J Clin Oncol. 1993;11:1466-1477.

24. Matthay KK, Shulkin B, Ladenstein R, et al. Criteria for evaluation of disease extent by ${ }^{123}$ I-metaiodobenzylguanidine scans in neuroblastoma: a report for the International Neuroblastoma Risk Group (INRG) Task Force. Br J Cancer. 2010; 102:1319-1326.

25. Youden WJ. Index for rating diagnostic tests. Cancer. 1950;3:32-35.

26. Pepe MS. The Statistical Evaluation of Medical Tests for Classification and Prediction. New York, NY: Oxford University Press; 2003.

27. Kaplan E, Meier P. Nonparametric estimation from incomplete observations. J Am Statist. 1958;53:457-481.

28. Peto R, Pike MC, Armitage P, et al. Design and analysis of randomized clinical trials requiring prolonged observation of each patient. II. Analysis and examples. Br J Cancer. 1977;35:1-39. 
29. Allison PD. Survival Analysis Using the SAS System: A Practical Guide. Cary, NC: SAS Institute Inc.; 1995.

30. Naranjo A, Parisi MT, Shulkin BL, et al. Comparison of ${ }^{123}$ I-metaiodobenzylguanidine (MIBG) and ${ }^{131}$ I-MIBG semi-quantitative scores in predicting survival in patients with stage 4 neuroblastoma: a report from the Children's Oncology Group. Pediatr Blood Cancer. 2011;56:1041-1045.

31. Ladenstein R, Lasset C, Hartmann O, et al. Impact of megatherapy on survival after relapse from stage 4 neuroblastoma in patients over 1 year of age at diagnosis: a report from the European Group for Bone Marrow Transplantation. J Clin Oncol. 1993;11:2330-2341.

32. Berthold F, Boos J, Burdach S, et al. Myeloablative megatherapy with autologous stem-cell rescue versus oral maintenance chemotherapy as consolidation treatment in patients with high-risk neuroblastoma: a randomised controlled trial. Lancet Oncol. 2005;6:649-658.

33. Frappaz D, Bonneu A, Chauvot P, et al. Metaiodobenzylguanidine assessment of metastatic neuroblastoma: observer dependency and chemosensitivity evaluation. The SFOP Group. Med Pediatr Oncol. 2000;34:237-241.

34. Matthay KK, Tan JC, Villablanca JG, et al. Phase I dose escalation of iodine131-metaiodobenzylguanidine with myeloablative chemotherapy and autologous stem-cell transplantation in refractory neuroblastoma: a new approaches to Neuroblastoma Therapy Consortium Study. J Clin Oncol. 2006;24: $500-506$.

35. Matthay KK, Yanik G, Messina J, et al. Phase II study on the effect of disease sites, age, and prior therapy on response to iodine-131-metaiodobenzylguanidine therapy in refractory neuroblastoma. J Clin Oncol. 2007;25:1054-1060.

36. Miano M, Garaventa A, Pizzitola MR, et al. Megatherapy combining I(131) metaiodobenzylguanidine and high-dose chemotherapy with haematopoietic progenitor cell rescue for neuroblastoma. Bone Marrow Transplant. 2001;27:571-574.

37. de Kraker J, Hoefnagel KA, Verschuur AC, et al. Iodine-131-metaiodobenzylguanidine as initial induction therapy in stage 4 neuroblastoma patients over 1 year of age. Eur J Cancer. 2008;44:551-556.

38. Gaze MN, Chang YC, Flux GD, et al. Feasibility of dosimetry-based high-dose ${ }^{131}$ I-meta-iodobenzylguanidine with topotecan as a radiosensitizer in children with metastatic neuroblastoma. Cancer Biother Radiopharm. 2005;20:195-199.

39. DuBois SG, Matthay KK. Radiolabeled metaiodobenzylguanidine for the treatment of neuroblastoma. Nucl Med Biol. 2008;35(suppl 1):S35-S48.

40. Yanik GA, Levine JE, Matthay KK, et al. Pilot study of iodine-131-metaiodobenzylguanidine in combination with myeloablative chemotherapy and autologous stem-cell support for the treatment of neuroblastoma. J Clin Oncol. 2002;20:2142-2149. 